\title{
Umgang mit Open-Access-Publikationsgebühren - Praxis und Perspektive in der Helmholtz-Gemeinschaft
}

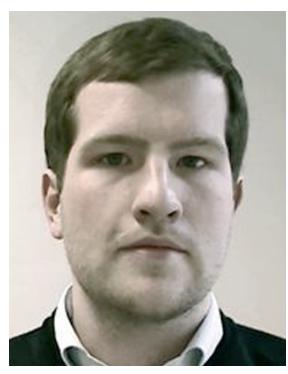

basis of surveys, recommendations and criteria for dealing with open access publication fees. These recommendations advise the Helmholtz Centres on the creation of open access publication funds at the organisational level. At the content level, the criteria give suggestions for dealing with open access publication fees.

Keywords: Open Access; Helmholtz Association; publication fees; journals

\section{Open Access in der Helmholtz- Gemeinschaft}

Die Helmholtz-Gemeinschaft ${ }^{1}$ vereint 17 naturwissenschaftlich-technische und medizinisch-biologische Forschungszentren. Die Helmholtz-Zentren leisten Beiträge zur Lösung großer und drängender Fragen von Gesellschaft, Wissenschaft und Wirtschaft durch eine strategisch-programmatisch ausgerichtete Spitzenforschung in sechs Forschungsbereichen: Energie, Erde und Umwelt, Gesundheit, Schlüsseltechnologien, Struktur der Materie sowie Luftfahrt, Raumfahrt und Verkehr.

Die Helmholtz-Gemeinschaft hat 2003 die ,Berliner Erklärung über den offenen Zugang zu wissenschaftlichem Wissen“ unterzeichnet und damit ihr Bestreben formuliert „die Weiterentwicklung des neuen Open Access-Paradigmas mit dem Ziel, den größtmöglichen Nutzen für Wissenschaft und Gesellschaft zu erreichen“" zu fördern. ${ }^{2}$

Die Berliner Erklärung bildet die Grundlage für vielfältige Open-Access-Aktivitäten, die an den Helmholtz-Zentren betrieben werden. 2004 hat die Mitgliederversammlung der Helmholtz-Gemeinschaft als erste deutsche wissenschaftliche Einrichtung eine Open-Access-Leitlinie verabschiedet. In dieser heißt es: „Publikationen aus der Helmholtz-Gemeinschaft sollen künftig ohne Ausnahme kostenlos zugänglich sein, soweit nicht ausdrückliche Vereinbarungen mit Verlagen und anderen dem entgegenstehen."

Zur Begleitung dieser Leitlinie wurde ein Arbeitskreis berufen, der die Open-Access-Aktivitäten der HelmholtzGemeinschaft lenkt. Dieses Gremium setzt sich aus Personen aus Wissenschaft, Wissenschaftsmanagement und Infrastruktureinrichtungen wie Bibliotheken, Daten- und Rechenzentren zusammen. 2005 formulierte der Arbeitskreis Open Access eine Roadmap zur Umsetzung des Open Access zu Textpublikationen und Forschungsdaten, die von der Mitgliederversammlung verabschiedet wurde. ${ }^{3}$

1 http://www.helmholtz.de.

2 Berliner Erklärung über den offenen Zugang zu wissenschaftlichem Wissen vom 22.10.2003, http://oa.mpg.de/lang/de/berlin-prozess/ berliner-erklarung/.

3 Realisierung des offenen Zugangs zu Publikationen und Daten aus der Helmholtz-Gemeinschaft, 01.02.2005, http://oa.helmholtz.de/fileadmin/upload/Vorschlag_Open_Access_Realisierung_2005-02-03.pdf. 
Zur Begleitung dieser Roadmap wurde ein Koordinationsbüro gegründet, welches die Zentren und ihre Mitarbeiter in Projektarbeit unter dem Label „Helmholtz Open Access Projekt" unterstützt. ${ }^{4}$ Diese Projektarbeit wird in einem engen Dialog mit den Open-Access-Beauftragten der Helmholtz-Zentren gemäß der Roadmap in vier Handlungsfeldern umgesetzt: Neben der Öffentlichkeitsarbeit rund um Open Access steht der Aufbau und die Vernetzung von Repositorien, die Förderung von Open-Access-Zeitschriften und der offene Zugang zu Forschungsdaten im Fokus. ${ }^{5}$ Workshops, Mailinglisten und ein Newsletter fördern die Information und Kommunikation aller Akteure. Das Koordinationsbüro agiert dabei als Serviceeinrichtung, die sich über die Projektarbeit hinaus um die Vernetzung und die Begleitung des Themas auf nationaler und internationaler Ebene kümmert.

Den Schwerpunkt bildeten zu Beginn der Open-AccessAktivitäten Aufbau und Vernetzung von Repositorien. Hier wurden die an den Helmholtz-Zentren betriebenen Publikationsdatenbanken zu Open-Access-Repositorien erweitert. Die Weiterentwicklung der Publikationsdatenbanken wird durch die Bibliotheken, Daten- und Rechenzentren im Rahmen des Informationsmanagements geleistet und ist zentrale Dienstleistung dieser Infrastruktureinrichtungen.

Durch Mitgliedschaften bei Open-Access-Zeitschriften fördern die Zentren die Entwicklung dieser Zeitschriften. So unterhält z.B. das Helmholtz-Zentrum DESY Mitgliedschaften in verschiedenen Open-Access-Zeitschriften im Bereich der Physik. ${ }^{6}$ Darüber hinaus sind Wissenschaftler der Helmholtz-Gemeinschaft als Herausgeber von Open-Access-Zeitschriften tätig. Weiter sind viele Zentren verlegerisch tätig. So betreiben beispielsweise das Forschungszentrum Jülich und das Karlsruher Institut für Technologie (KIT) eigene Verlage, deren Publikationen Open Access erscheinen. ${ }^{7}$ Aufgrund der rasanten Entwicklungen im Feld der OpenAccess-Zeitschriften hat der Arbeitskreis Open Access der Helmholtz-Gemeinschaft 2010 eine Fokussierung auf dieses Thema beschlossen. ${ }^{8}$

Im Bereich des offenen Zugangs zu Forschungsdaten sind vielfältige Aktivitäten zu nennen: Herauszuheben ist der Betrieb von Weltdatenzentren ${ }^{9}$ an den Zentren der Helm-

4 http://oa.helmholtz.de.

5 Ausführliche Darstellungen finden sich bei Pampel, H.: Open Access in der Helmholtz-Gemeinschaft Status und Perspektiven. In: Tagungsband 2009 der Arbeitsgemeinschaft der Spezialbibliotheken. Preprint online: http://edoc.gfz-potsdam.de/gfz/13878 sowie bei Bertelmann, R.; Hübner, A.: Open Access im Dienst der Wissenschaft: Umsetzung von freiem Zugang in der Helmholtz-Gemeinschaft. In: Zeitschrift für Bibliothekswesen und Bibliographie 54 (2007), Nr. 4/5, S. 246-250, http://edoc.gfz-potsdam.de/gfz/10399.

6 Siehe dazu die Informationen auf der Website der Bibliothek des DESY: http://library.desy.de/open_access/.

7 Siehe dazu Verlag des Forschungszentrums Jülich: http://www2. fz-juelich.de/zb/verlag und KIT Scientific Publishing: http://www. ksp.kit.edu.

8 Siehe dazu die folgenden Kapitel.

9 Im Rahmen des World Data System (WDS) des International Council for Science (ICSU). Siehe hierzu die Website des WDS: http://www. icsu-wds.org.
holtz-Gemeinschaft. ${ }^{10}$ Diese Datenzentren widmen sich der dauerhaften Zugänglichkeit von Forschungsdaten. Neben weiteren vielfältigen disziplinären Aktivitäten sind die Zentren in interdisziplinäre Aktivitäten rund um die dauerhafte Zugänglichkeit von Forschungsdaten eingebunden: auf nationaler Ebene beispielsweise im Rahmen des DFG-Projekts „Rahmenbedingungen einer disziplinübergreifenden Forschungsdateninfrastruktur" ${ }^{\text {11 }}$, auf europäischer Ebene z.B. durch die EU-Projekte Alliance Permanent Access to the Records of Science in Europe Network (APARSEN) ${ }^{12}$, European Collaborative Data Infrastructure (EUDAT) ${ }^{13}$ und Opportunities for Data Exchange (ODE). ${ }^{14}$

\section{Herausforderung Open-Access- Publikationsgebühren}

Die Landschaft der Open-Access-Zeitschriften entwickelt sich dynamisch. Seit 2000 beträgt die durchschnittliche Wachstumsrate pro Jahr im Bereich des Zeitschriftenmarktes 18 Prozent. ${ }^{15}$ Das Directory of Open Access Journals (DOAJ) verzeichnete im September 20117.000 Open-Access-Zeitschriften. ${ }^{16}$ Open Access gewinnt für kommerzielle Verlage deutlich an Relevanz und ist somit längst nicht mehr nur eine Publikationsstrategie wissenschaftlicher Institutionen und Fachgesellschaften.

Unter dem Namen SpringerOpen hat der Wissenschaftsverlag Springer Mitte 2010 ein disziplinübergreifendes Open-Access-Programm aufgelegt und damit ein deutliches Zeichen für Open Access gesetzt. ${ }^{17}$ SpringerOpen wird in enger Zusammenarbeit mit dem Open-Access-Verlag Bio-

10 Das World Data Center for Marine Environmental Sciences (WDCMARE), welches vom Alfred-Wegener-Institut für Polar- und Meeresforschung (AWI) und vom Zentrum für Marine Umweltwissenschaften der Universität Bremen (MARUM) betrieben wird, und das World Data Center for Remote Sensing of the Atmosphere (WDC- RSAT) des Deutschen Zentrums für Luft- und Raumfahrt (DLR). Darüber hinaus sind das Alfred-Wegener-Institut für Polar- und Meeresforschung und das Helmholtz-Zentrum Geesthacht (HZG) Gesellschafter des Deutschen Klimarechenzentrums (DKRZ), welches das World Data Center for Climate betreibt. Die inzwischen weltweit verbreitete $\mathrm{Pu}$ blikation von Forschungsdaten mit Hilfe von Digital Object Identifier (DOI) wurde von diesen Datenzentren im Rahmen des DFG-Projektes STD-DOI konzipiert. Siehe dazu: http://www.std-doi.de.

11 http://www.forschungsdaten.org. An diesem Projekt ist das Helmholtz-Zentrum Potsdam Deutsches GeoForschungsZentrum GFZ beteiligt.

12 http://www.aparsen.eu. An diesem Projekt ist das Alfred-WegenerInstitut für Polar- und Meeresforschung beteiligt.

13 http://www.eudat.eu. An diesem Projekt sind das Forschungszentrum Jülich und das Karlsruher Institut für Technologie (KIT) beteiligt.

14 http://www.ode-project.eu. An diesem Projekt ist das Alfred-Wegener-Institut für Polar- und Meeresforschung beteiligt.

15 Laakso, M., Welling, P., Bukvova, H., Nyman, L., Björk, B.-C., und Hedlund, T.: The development of open access journal publishing from 1993 to 2009. In: PloS one (2011), 6(6), e20961. http://dx.doi. org/10.1371/journal.pone.0020961.

16 Siehe Pressemitteilung vom 15.09.2011 unter http://www.doaj.org/ doaj?func=loadTempl\&templ $=110915 \&$ uiLanguage $=e n$.

17 http://www.springeropen.com. Siehe hierzu auch: Goerner, B.: Die Zeit ist reif - Springer entwickelt Open Access weiter. In: B.I.T.online 13 (2010), Nr. 4, S. 384-385, http://www.b-i-t-online.de/heft/201004-schwerpunkt3.pdf. 
Med Central ${ }^{18}$ betrieben, der 2008 von Springer übernommen wurde. Rund ein Jahr nach Auftakt des Programms gab der Verlag im Juni 2011 den Start des fünfzigsten SpringerOpen-Journals bekannt. 2011 zog Wiley nach und kündigte im Februar ein Open-Access-Programm unter der Marke Wiley Open Access an. Im Oktober 2011 hatte der Verlag vier Open-Access-Zeitschriften im Portfolio. ${ }^{19}$

Darüber hinaus haben Verlage wie Britisch Medical Journal, Nature Publishing Group und Royal Society Publishing im Jahr 2011 Open-Access-Zeitschriften gegründet. Interessant ist, dass für diese Neugründungen häufig das Konzept der Zeitschrift PLoS ONE der Public Library of Science $(\mathrm{PLoS})^{20}$ als Vorbild dient. Im Juni 2011 erschien der erste Aufsatz in der Zeitschrift Scientific Reports ${ }^{21}$ der Nature Publishing Group. Wie bei PLoS ONE steht bei Scientific Reports die schnelle Veröffentlichung und die hohe Sichtbarkeit eines Aufsatzes ohne Beschränkung auf eine Disziplin im Vordergrund. ${ }^{22}$ Der Begutachtungsprozess fokussiert die Qualität einer Publikation, „Neuigkeitswert“ oder „Einzigartigkeit" spielen eine nachgeordnete Rolle. Dieses Konzept wird auch unter disziplinärem Fokus übernommen: Die Royal Society Publishing beispielsweise kündigte im September 2011 den Start der Open-Access-Zeitschrift Open Biology an. ${ }^{23}$ Diese Zeitschrift ist die erste originäre OpenAccess-Zeitschrift der Royal Society. Wie auch PLoS ONE wirbt die Zeitschrift mit einem schnellen Review-Prozess. ${ }^{24}$

Mit dem Wachstum des Open-Access-Zeitschriftenmarktes gewinnt die Diskussion über die Geschäftsmodelle der Open-Access-Zeitschriften an Bedeutung. Die Geschäftsmodelle können in gebührenbasierte und nicht-gebührenbasierte Modelle unterteilt werden. Während sich im Rahmen nicht-gebührenbasierter Modelle Zeitschriften beispielsweise durch eine herausgebende Institution finanzieren, steht im Bereich der gebührenbasierten Modelle die Finanzierung über Open-Access-Publikationsgebühren im Vordergrund. Laut einer Erhebung von Shieber aus dem Jahr 2009 erheben nur 27 Prozent der im DOAJ gelisteten Zeitschriften Publikationsgebühren. ${ }^{25}$ In den naturwissenschaftlichen Disziplinen, in denen die Zentren der Helmholtz-Gemeinschaft forschen,

18 http://www.biomedcentral.com.

19 http://www.wileyopenaccess.com.

20 http://www.plosone.org.

21 http://www.nature.com/srep.

22 Vergleiche hierzu beispielsweise die Selbstbeschreibung der Zeitschriften: PLoS ONE unter http://www.plosone.org/static/information. action, Scientific Reports unter http://www.nature.com/srep/about sowie die lesenswerten Blogbeiträge von Fenner und Neylon: Fenner, M.: New journal "Nature ONE" launched today. In: Gobbledygook, 06.01.2011, http://blogs.plos.org/mfenner/2011/01/06/new-journalnature-one-launched-today/. Neylon, C.: PLoS (and NPG) redefine the scholarly publishing landscape. In: Science in the Open, 07.01.2011, http://cameronneylon.net/blog/plos-and-npg-redefine-the-scholarlypublishing-landscape/.

23 http://rsob.royalsocietypublishing.org.

24 Siehe dazu die Beschreibung der Zeitschrift unter http://rsob. royalsocietypublishing.org/site/misc/about.xhtml.

25 Shieber, S.: What percentage of open-access journals charge publication fees? In: The Occasional Pamphlet, 29.03.2009, http://blogs. law.harvard.edu/pamphlet/2009/05/29/what-percentage-of-open-access-journals-charge-publication-fees/. dominiert jedoch das Geschäftsmodell der Publikationsgebühr. Dieses wurde insbesondere durch die Verlage Public Library of Science (PLoS) und BioMed Central professionalisiert und wird häufig unter dem Begriff ,author pays“ diskutiert. Dieser Begriff suggeriert fälschlicherweise, dass ein Wissenschaftler als Privatperson für die entstehenden Kosten aufkommt. Üblicherweise werden diese Kosten jedoch von einer Förderorganisation oder der Institution des Publizierenden übernommen.

Einige Verlage, die das Geschäftsmodell der Publikationsgebühren betreiben, bieten sogenannte ,institutionelle Mitgliedschaften" an. Hier zahlt eine wissenschaftliche Institution eine Jahresgebühr, die entweder alle Kosten für die Publikationen ihrer Institutionsangehörigen abdeckt, oder aber als Grundlage für einen prozentualen Rabatt auf die Publikationsgebühren dient.

Viele Forschungsförderer unterstützen die frei zugängliche Veröffentlichung von Ergebnissen aus geförderten Projekten. Dazu werden Mittel zur Verfügung gestellt, die Autoren bei der Publikation in einer sich durch Publikationsgebühren finanzierenden Open-Access-Zeitschrift in Anspruch nehmen können. So stellen beispielsweise die Deutsche Forschungsgemeinschaft und die Europäische Kommission im Rahmen ihrer Forschungsförderung Mittel zur Finanzierung von Open-Access-Publikationsgebühren bereit. Wollen Autoren eines Projekts, das unter den OpenAccess-Piloten des siebten Forschungsrahmenprogramms fällt, in einer Open-Access-Zeitschrift publizieren, die $\mathrm{Pu}$ blikationsgebühren erhebt, können sie sich diese Gebühren erstatten lassen. ${ }^{26}$

Im Rahmen der Study of Open Access Publishing (SOAP) haben sich rund 90 Prozent von 3.000 in Deutschland tätigen Wissenschaftlern positiv zu Open Access geäußert. Eine der zentralen Herausforderungen, um Wissenschaftlern die Publikation in Open-Access-Zeitschriften zu ermöglichen, ist ein organisierter Umgang mit anfallenden Publikationsgebühren. Die SOAP-Studie zeigt, dass die Finanzierung von Publikationsgebühren für in Deutschland tätige Wissenschaftler im internationalen Vergleich weniger problematisch ist; dennoch beurteilen rund 40 Prozent der Befragten die Finanzierung als problematisch. ${ }^{27}$

Vor dem Hintergrund der dynamischen Entwicklungen auf dem Zeitschriftenmarkt sind wissenschaftliche Institutionen weltweit bestrebt, ihren Wissenschaftlern den Umgang mit diesen Gebühren so einfach wie möglich zu machen und damit die Entwicklung von Open Access zu fördern.

Internationale Beachtung fand 2009 die Initiierung eines „Compact for Open Access Publishing Equity“ (COPE) durch fünf US-Universitäten. In diesem Statement erklären die Unterzeichner ihren Willen, dauerhafte Mechanismen zur Übernahme für angemessene Publikationsgebühren zu

26 Siehe dazu z.B. die weiterführenden Informationen auf der Website der Europäischen Kommission: http://ec.europa.eu/research/s cience-society/open_access/.

27 Dallmeier-Tiessen, S.; Lengenfelder, A.: Open Access in der deutschen Wissenschaft - Ergebnisse des EU-Projekts ,,Study of Open Access Publishing“ (SOAP). In: GMS Medizin-Bibliothek-Information 2011, 11(1-2):Doc03, http://dx.doi.org/10.3205/mbi000218. 
schaffen..$^{28}$ Aktuell implementieren wissenschaftliche Institutionen weltweit sogenannte Open-Access-Publikationsfonds zur Finanzierung von Publikationsgebühren, die bei einer Publikation in Open-Access-Zeitschriften anfallen. Prominentes Beispiel ist die Harvard University: Unter dem Titel „Harvard Open-Access Publishing Equity (HOPE) “29 stehen Wissenschaftlern Mittel zur Open-Access-Veröffentlichung zur Verfügung. Nach der Scholarly Publishing and Academic Resources Coalition (SPARC) wird ein Open-Access-Publikationsfonds wie folgt definiert: ,a pool of money set aside by an institution or other research-sponsoring entity specifically to defray or cover processing fees for articles published by members of the institution in open-access journals. While open-access funds are administered in a variety of fashions with a wide array of policy distinctions, they share a common goal of encouraging researchers to make their work open to the public. " ${ }^{30}$

Die deutschen Wissenschaftsorganisationen haben sich im Rahmen ihrer 2008 gestarteten Schwerpunktinitiative „Digitale Information“ u.a. darauf verständigt, im Bereich der Open-Access-Zeitschriften die „Weiterentwicklung von Geschäfts- und Fördermodellen und deren gemeinschaftliche Finanzierung " zu fördern. ${ }^{31}$ So kündigte die Deutsche Forschungsgemeinschaft (DFG) 2009 das Förderprogramm „Open-Access-Publizieren“ an. ${ }^{32}$ Im Rahmen des Programms können Hochschulen Mittel beantragen, um Publikationen ihrer Wissenschaftler in originären Open-Access-Zeitschriften zu finanzieren. ${ }^{33}$ Dabei müssen 25 Prozent der beantragten Mittel durch die Hochschule selbst aufgebracht werden. Die Förderung zielt auf den Aufbau von sogenannten OpenAccess-Publikationsfonds ab. Die Mittel der Publikationsfonds, welche häufig von Bibliotheken verwaltet werden, können von Wissenschaftlern bei der Publikation in einer Open-Access-Zeitschrift beantragt werden. Im Jahr 2010 haben zwölf Hochschulen Mittel aus dem DFG-Programm eingeworben, darunter auch das Karlsruher Institut für Technologie (KIT). ${ }^{34}$

28 http://www.oacompact.org. Zum Hintergrund des COPE siehe: Shieber, S. M.: Equity for Open-Access Journal Publishing. In: PLoS Biolgy 2009, 7(8): e1000165. http://dx.doi.org/10.1371/journal. pbio. 1000165 .

29 http://osc.hul.harvard.edu/hope.

30 Scholarly Publishing and Academic Resources Coalition (SPARC): Campus-based open-access publishing funds: frequently asked questions. Online unter http://www.arl.org/sparc/openaccess/funds/faq. shtml. Siehe hierzu auch: Eckman, C. D., Weil, B.T.: Institutional Open Access Funds: Now Is the Time. In: PLoS Biology (2010), 8(5): e1000375. Online: http://dx.doi.org/10.1371/journal.pbio.1000375.

31 Siehe dazu: Allianz der Wissenschaftsorganisationen: Leitbild der Schwerpunktinitiative „Digitale Information“, 2008, http://www. allianz-initiative.de/fileadmin/user_upload/keyvisuals/atmos/pm_ allianz_digitale_information_details_080612.pdf.

32 Deutsche Forschungsgemeinschaft: Merkblatt Open Access Publizieren, 2010, http://www.dfg.de/download/programme/ wissenschaftliche_literaturversorgung_informationssysteme/antragstellung/12_20/12_20.pdf.

33 So genannte hybride Open-Access-Geschäftsmodelle werden nicht unterstützt.

34 Siehe dazu: DFG: Information für die Wissenschaft Nr. 1, 04.01.2011, http://www.dfg.de/foerderung/info_wissenschaft/archiv/2011/info_ wissenschaft_11_01/index.html, sowie die Website des Karlsruher
Während sich in der Vergangenheit Mechanismen zur Finanzierung von Subskriptionsgebühren etabliert haben, stehen diese im Umgang mit Publikationsgebühren noch aus. Grundfrage bei dem Aufbau eines Publikationsfonds ist die Finanzierung dieses Instrumentes. Darüber hinaus müssen Kriterien geschaffen werden, die festlegen, welche Publikationen durch den Fonds finanziert werden können.

\section{Praxis in der Helmholtz-Gemeinschaft}

Vor dem Hintergrund der beschriebenen Entwicklungen hat sich der Arbeitskreis Open Access der Helmholtz-Gemeinschaft Anfang 2010 des Themas angenommen. In einem ersten Schritt wurden die aktuellen Praktiken im Umgang mit Open-Access-Publikationsgebühren unter die Lupe genommen.

In einer Erhebung der Arbeitsgruppe Open Access in der Allianz der deutschen Wissenschaftsorganisationen wurden 2010 außeruniversitäre Forschungseinrichtungen und Hochschulen zum Umgang mit Publikationsgebühren befragt. ${ }^{35}$ In der Helmholtz-Gemeinschaft wurden diese Fragen durch die Open-Access-Beauftragten der Zentren bearbeitet.

Die Ergebnisse der Umfragen zeigen, dass sich die Zentren des Themas angenommen haben. Publikationsgebühren, die bei der Veröffentlichung in Open-Access-Zeitschriften anfallen, werden durch die Zentren übernommen. Die Ergebnisse machen jedoch auch die Herausforderungen bei dem Umgang mit Open-Access-Publikationsgebühren deutlich.

Eine dieser Herausforderungen ist die Organisation der Open-Access-Publikationsgebühren in einer Institution. Die Ergebnisse der Allianz-Umfrage zeigen, dass es in der Helmholtz-Gemeinschaft unterschiedliche Verfahren im Umgang mit den Publikationsgebühren gibt. Grund dafür ist in erster Linie die Selbstständigkeit der einzelnen Forschungszentren. Während z.B. am Forschungszentrum Jülich Open-AccessPublikationsgebühren wie auch „klassische“ Publikationsgebühren (z.B. so genante ,pages charges" und ,color charges") von der Zentralbibliothek des Forschungszentrums verwaltet werden, werden z.B. am Max-Delbrück-Centrum für Molekulare Medizin (MDC) nur Open-Access-Publikationsgebühren der Springer-Programme BioMed Central und SpringerOpen durch die Bibliothek verwaltet. Open-AccessPublikationsgebühren wie auch „,klassische“ Publikationsgebühren, die bei der Veröffentlichung außerhalb der beiden Springer-Programme anfallen, werden durch die einzelnen wissenschaftlichen Abteilungen des MDC getragen. Diese beiden exemplarisch genannten Praktiken zweier HelmholtzZentren stehen stellvertretend für vielfältige Verfahren beim Umgang mit Publikationsgebühren.

Institut für Technologie (KIT) mit weiterführenden Informationen: http://www.bibliothek.kit.edu/cms/kit-publikationsfonds.php.

35 Die Umfrage wurde durch das Leibniz-Institut für Sozialwissenschaften (GESIS) wissenschaftlich betreut. Eine Gesamtanalyse ist in Arbeit. Die Datenerhebung erfolgte in Form eines standardisierten Online-Fragebogens. An der Umfrage haben 12 von 16 HelmholtzZentren teilgenommen. Das entspricht einer Rücklaufquote von 75 Prozent. 
Auf Basis der erwähnten Umfrage-Ergebnisse lassen sich in der Helmholtz-Gemeinschaft drei Modelle charakterisieren: Beim ersten Modell verwaltet die Bibliothek eines Zentrums die Mittel, die für die Finanzierung von OpenAccess-Publikationsgebühren verwendet werden; beim zweiten Modell verwaltet die Bibliothek einen Teil der Mittel; beim dritten Modell werden die Mittel ausnahmslos durch die jeweiligen wissenschaftlichen Organisationseinheiten verwaltet. Das zweitgenannte Modell ist das verbreitetste. Hintergrund sind vertragliche Vereinbarungen der Zentren mit Verlagen über Open-Access-Programme. Die Höhe der jährlichen Ausgaben für Open-Access-Publikationsgebühren erreicht laut Umfrage pro Zentrum bis zu 80.000 Euro.

Die Ergebnisse der Online-Umfrage machen aber auch die zukünftigen Herausforderungen bei der Organisation der Mittel für Open-Access-Publikationsgebühren deutlich: In der Mehrheit der Zentren gibt es bisher kein festgelegtes Budget für Open-Access-Publikationsgebühren. Dies zeigt, dass die Erfahrungen im Umgang mit solchen Kosten in vielen Zentren noch zu gering sind, um ein Budget im Voraus festzulegen. Erfreulich ist, dass sich die Zentren des Themas annehmen und bestrebt sind, institutionelle Workflows zu schaffen, um den Autoren einen möglichst unkomplizierten Umgang mit den Gebühren zu ermöglichen.

Neben diesen organisatorischen Aspekten widmete sich die Umfrage auch inhaltlichen Kriterien, nach denen die Übernahme von Open-Access-Publikationsgebühren an einer Einrichtung geregelt ist: Für den Umgang mit hybriden Zeitschriften gibt es keine einheitliche Lösung in den Zentren. Einige übernehmen die Kosten auch bei hybriden Zeitschriften, andere übernehmen sie nicht. Verlag und Impact Factor einer Zeitschrift werden bei der Entscheidung über die Vergabe der Open-Access-Publikationsgebühren mehrheitlich nicht berücksichtigt.

Neben dem Blick auf die Praxis der Zentren hat das Helmholtz Open Access Koordinationsbüro 2010 Wissenschaftler, die dem Herausgebergremium einer OpenAccess-Zeitschrift angehören, zum Thema befragt. Auf Basis von Recherchen auf den Webseiten einiger relevanter OpenAccess-Verlage wurden rund 100 Personen identifiziert, die an einem Helmholtz-Zentrum forschen und sich darüber hinaus als Editor einer Open-Access-Zeitschrift engagieren. Die Ergebnisse dieser Umfrage sind keinesfalls repräsentativ, werfen jedoch einige Schlaglichter auf die Position von Wissenschaftlern, die mit Open Access vertraut sind. Wenig überraschend gab es in der Umfrage viele Parallelen zu der erwähnten SOAP-Studie. Zusammenfassend lässt sich feststellen, dass die Forderung nach einfachen Mechanismen im Umgang mit Open-Access-Publikationsgebühren und eine stärkere Berücksichtigung von Open-Access-Publikationen im Rahmen von Evaluierungen zwei zentrale Anliegen der Editoren sind.

Über diese beiden Umfragen hinaus hat das Koordinationsbüro 2010 und 2011 mehrere Diskussionen mit den Open-Access-Beauftragten der Zentren zum Thema geführt. Einen Schwerpunkt dieser Diskussionen bildete die Herausforderung des Umgangs mit hybriden Open-Access-Angebo- ten. Während die Mehrzahl der Zentren diesem Modell mit Blick auf die Gefahr des sogenannten ,double dippings““36 skeptisch bis ablehnend gegenübersteht, gibt es seitens der Wissenschaft ein steigendes Interesse an der Open-AccessPublikation in hybriden Zeitschriften. Aufgrund dieser unbefriedigenden Situation im Umgang mit hybriden Zeitschriften wird sich das Helmholtz Open Access Projekt diesem Thema in Zukunft verstärkt widmen.

Begleitet durch das Koordinationsbüro der HelmholtzGemeinschaft haben die Bibliotheken der Zentren 2010 einen Konsortialvertrag mit Springer über das Open-AccessProgramm SpringerOpen geschlossen. Ein weiterer Vertrag wurde 2011 mit dem Institute of Physics über die Publikationsgebühren des New Journal of Physics (NJP) vereinbart. Bereits in der Vergangenheit wurden im Rahmen sogenannter institutioneller Mitgliedschaften Verträge über Open-AccessPublikationsgebühren durch einzelne Zentren abgeschlossen (z.B. mit BioMed Central). Mit den Konsortialverträgen konnten nun auf zentrumsübergreifender Ebene optimale Konditionen für alle Zentren geschaffen werden. Darüber hinaus haben die beiden Konsortialverträge die Diskussion über den Umgang mit Open-Access-Publikationsgebühren in der Helmholtz-Gemeinschaft gefördert und die Weichen für zukünftige Verhandlungen mit Verlagen gestellt.

\section{Perspektiven im Umgang mit Open-Access-Publikationsgebühren}

Um Open Access zielgerichtet zu fördern, ist es nötig, $\mathrm{Au}-$ toren einfache Verfahren und Mechanismen zur Übernahme von Open-Access-Publikationsgebühren anzubieten. Der Arbeitskreis Open Access in der Helmholtz-Gemeinschaft hat auf Basis der beschriebenen Erhebungen und Diskussionen 2011 eine Empfehlung zum Thema verabschiedet. In dieser heißt es: „,Der Arbeitskreis Open Access in der Helmholtz-Gemeinschaft empfiehlt den Zentren, zur Förderung von Open Access geeignete Mechanismen zur Übernahme von Publikationsgebühren aufzubauen. Über diese Publikationsfonds, die von den Bibliotheken verwaltet werden, sollen Veröffentlichungen in Open-Access-Zeitschriften finanziert werden, für die keine Drittmittel bereitstehen. Die Finanzierung und die Steuerung der Open-Access-Publikationsfonds müssen in die jeweiligen Strukturen der Zentren integriert sein. Eine zentrale Rolle kommt dabei den Bibliotheken der Helmholtz-Zentren zu.“

Zur Umsetzung dieser Empfehlung wurden in der Helmholtz-Gemeinschaft Kriterien erarbeitet, die bei dem Betrieb von Publikationsfonds berücksichtigt werden sollten. Dabei sollen in Anlehnung an das Förderprogramm „Open-AccessPublizieren“ der Deutschen Forschungsgemeinschaft (DFG) u.a. folgende Punkte beachtet werden:

36 Siehe dazu z.B. Kämper, B.-C.: Hybrid publizieren, doppelt abkassieren ... In: Archivalia, 27.10.2009 sowie die Ergänzung vom 05.11.2009, http://archiv.twoday.net/stories/6013528/. 
Ein Angehöriger des Zentrums muss als ,,submitting author" oder als ,,corresponding author" an dem Artikel beteiligt sein.

Die Qualität der Zeitschrift muss durch die im jeweiligen Fach anerkannten Qualitätssicherungsverfahren gesichert sein.

Die Zeitschrift sollte im Directory of Open Access Journals (DOAJ) gelistet sein.

Falls die Publikation in einem kommerziell arbeitenden Verlag erscheint, sollte dieser Mitglied in der Open Access Scholarly Publishers Association (OASPA) ${ }^{37}$ sein und deren Kriterien entsprechen.

Der Artikel sollte in einer Zeitschrift erscheinen, die unter der Creative-Commons-Lizenz „Namensnennung“"erscheint.

So genannte hybride Open-Access-Modelle (z.B. Springer Open Choice oder Elsevier Sponsored Articles) sollten nicht unterstützt werden.

Neben diesen inhaltlichen Kriterien werden auch einige organisatorische Empfehlungen gegeben. So wird beispielsweise angeregt, bei der Einrichtung des Fonds pro Organisationseinheit eine Summe zu definieren, die in Anspruch genommen werden kann, um die unterschiedlichen Publikationskulturen der Organisationseinheit zu berücksichtigen. Darüber hinaus wird empfohlen, die Publikationsfonds jährlich zu evaluieren.

\section{$5 \quad$ Fazit}

Wie dargestellt, gewinnt die Bedeutung des organisierten Umgangs mit Publikationsgebühren an Relevanz. Weltweit beginnen wissenschaftliche Institutionen Mechanismen zu entwickeln, um den Umgang mit Publikationsgebühren für Wissenschaftler transparent und für ihre Organisation nachhaltig zu gestalten. Vermehrt verwalten wissenschaftliche Institutionen unter dem Schlagwort „Publikationsfonds“ Mittel, die von Autoren bei der Veröffentlichung in OpenAccess-Zeitschriften verwendet werden können.

Mit Blick auf die praktische Umsetzung sind vielfältige Herausforderungen zu nennen, die die Diskussion zum Thema in den kommenden Jahren prägen werden. Der Blick auf die komplexe Publikationslandschaft der Open-AccessZeitschriften (die z.B. neben kommerziellen Verlagen durch eine Vielzahl von Fachgesellschaften und wissenschaftlichen Einrichtungen, die verlegerisch tätig sind, geprägt ist) macht deutlich, dass der Umgang mit diesen Publikationsgebühren in Zukunft weiter professionalisiert werden muss. So ist beispielsweise bei jeder Veröffentlichung, bei der Publikationsgebühren anfallen, zu klären, ob durch Drittmittelförderung Mittel zur Finanzierung der Publikationsgebühren in Anspruch genommen werden können.

Neben solchen organisatorischen Fragen stehen vermehrt inhaltliche Fragen im Fokus. Beispiel: Im Bereich der Lizenzierung der Inhalte setzen bedauerlicherweise nicht alle Open-Access-Zeitschriften auf die Creative-Commons-Lizenz „Namensnennung“ und behindern durch die Verwen-

37 http://www.oaspa.org. dung stärker einschränkender Lizenzen z.B. die Nutzung von Text-Mining-Verfahren durch Partner in der Wirtschaft. Das Anliegen, von Open Access einen ,re-use“ der Inhalte zu ermöglichen, wird damit behindert. Für wissenschaftliche Institutionen und Förderer stellt sich hier die Frage, wie mit solchen Publikationsmodellen umgegangen werden soll und ob eine Förderung dieser Zeitschriften im Sinn von Open Access ist. ${ }^{38}$

Die Helmholtz-Gemeinschaft hat sich dieser Fragen angenommen und fördert im Dialog mit nationalen und internationalen Wissenschaftsorganisationen die Diskussion zum Thema.

Auf Ebene der Helmholtz-Zentren lassen sich neue Maßnahmen und Verfahren im Umgang mit Publikationsgebühren feststellen. Eine Empfehlung und begleitende Kriterien unterstützen die Weiterentwicklung von internen Mechanismen des Umgangs mit Open-Access-Publikationsgebühren. Zukünftige Entwicklungen im Bereich der Open-AccessZeitschriften werden immer wieder Anpassungen der Aktivitäten erfordern.

Die Helmholtz-Gemeinschaft wird die dynamische Entwicklung von Open Access weiter fördern mit dem Ziel, die in an den Zentren gewonnenen Erkenntnisse Gesellschaft, Wissenschaft, Wirtschaft und Politik zugänglich und nachnutzbar zu machen.

\section{Danksagung}

Die Verfasser des Beitrags danken den Mitgliedern des Arbeitskreises Open Access für anregende Diskussionen zum Thema.

\section{Referenzen}

Bertelmann, R.; Hübner, A.: Open Access im Dienst der Wissenschaft: Umsetzung von freiem Zugang in der Helmholtz-Gemeinschaft. In: Zeitschrift für Bibliothekswesen und Bibliographie 54 (2007), Nr. 4/5, S. 246-250, http:// edoc.gfz-potsdam.de/gfz/10399.

Dallmeier-Tiessen, S.; Lengenfelder, A.: Open Access in der deutschen Wissenschaft - Ergebnisse des EUProjekts „Study of Open Access Publishing“ (SOAP). In: GMS Medizin - Bibliothek - Information 2011, 11(1-2):Doc03, http://dx.doi.org/10.3205/mbi000218.

Eckman, C. D., Weil, B.T.: Institutional Open Access Funds: Now Is the Time. In: PLoS Biology (2010), 8(5): e1000375. Online: http://dx.doi.org/10.1371/journal. pbio. 1000375 .

Eisen, M.: PLoS Won. In: it is NOT junk, 25.10.2011, http:// www.michaeleisen.org/blog/?p=686.

38 Siehe hierzu z.B. Murray-Rust, P.: Suboptimal/missing Open Licences by Wiley and Royal Society. In: petermr's blog, 27.10.2011, http:// blogs.ch.cam.ac.uk/pmr/2011/10/27/suboptimalmissing-open-licences-by-wiley-and-royal-society/. Eisen, M.: PLoS Won. In: it is NOT junk, 25.10.2011, http://www.michaeleisen.org/blog/?p=686. 
Goerner, B.: Die Zeit ist reif - Springer entwickelt Open Access weiter. In: B.I.T.online 13 (2010), Nr. 4, S. 384385, http://www.b-i-t-online.de/heft/2010-04-schwerpunkt3.pdf .

Kämper, B.-C.: Hybrid publizieren, doppelt abkassieren ... In: Archivalia, 27.10.2009 sowie die Ergänzung vom 05.11.2009, http://archiv.twoday.net/stories/6013528/.

Laakso, M., Welling, P., Bukvova, H., Nyman, L., Björk, B.-C., und Hedlund, T.: The development of open access journal publishing from 1993 to 2009. In: PloS one (2011), 6(6), e20961. http://dx.doi.org/10.1371/journal. pone.0020961.

Murray-Rust, P.: Suboptimal/missing Open Licences by Wiley and Royal Society. In: petermr's blog, 27.10.2011, http:// blogs.ch.cam.ac.uk/pmr/2011/10/27/suboptimalmissingopen-licences-by-wiley-and-royal-society/.

Pampel, H.: Open Access in der Helmholtz-Gemeinschaft Status und Perspektiven. In: Tagungsband 2009 der Arbeitsgemeinschaft der Spezialbibliotheken. Preprint online: http://edoc.gfz-potsdam.de/gfz/13878

Shieber, S.: What percentage of open-access journals charge publication fees? In: The Occasional Pamphlet, 29.03.2009, http://blogs.law.harvard.edu/pamphlet/ 2009/05/29/what-percentage-of-open-access-journalscharge-publication-fees/.

Shieber, S. M.: Equity for Open-Access Journal Publishing. In: PLoS Biolgy 2009, 7(8): e1000165. http://dx.doi. org/10.1371/journal.pbio.1000165. 